\title{
O panorama da Pós-graduação Stricto Sensu em Turismo no Brasil a partir do perfil docente
}

\author{
Fausi Kalaoum* Mariana Lopes** Mariana Tomazin*** \\ Universidade de São Paulo (Brasil)
}

\begin{abstract}
Resumo: O objetivo desse artigo constitui-se em investigar o universo da pós-graduação stricto sensu em Turismo no Brasil a partir dos cursos oferecidos atualmente e acerca do perfil dos docentes no âmbito dos programas que pertencem. O universo da pesquisa contemplou 10 programas de pós-graduação do território brasileiro, totalizando a caracterização de 131 docentes. A pesquisa utilizou-se de uma abordagem mista, contemplando aspectos qualitativos e quantitativos, de maneira complementar. Realizou-se revisão bibliográfica sobre o percurso histórico dos programas de pós-graduação e da produção do conhecimento na área no Brasil. A coleta de dados adotou uma postura de varredura a partir das informações disponíveis na Plataforma Lattes. Para as análises estatísticas fez-se o uso do software SPSS statistics e microsoft excel e para análise qualitativa utilizou-se o recurso visual de nuvem de palavras. O trabalho contribui para o debate da relação entre pós-graduação e produção científica do turismo no caso brasileiro.
\end{abstract}

Palavras-chave: Pós-Graduação em Turismo; Docentes em Turismo; Produção do Conhecimento; Brasil.

The panorama of the Stricto Sensu Tourism Postgraduate in Brazil from the profile's Professors

Abstract: The aim of this article is to investigate the universe of stricto sensu postgraduate studies in Tourism in Brazil based on the courses currently offered and on the profile of professors in the scope of the programs to which they belong. The universe included 10 postgraduate programs in Tourism and related areas of the Brazilian territory, totaling the characterization of 131 professors. The research used a mixed approach, contemplating qualitative and quantitative aspects, in a complementary way. A bibliographic review on the theme was carried out in order to support the understanding of the historical path of postgraduate programs and the production of knowledge in the area, in Brazil. The data collection adopted a scanning attitude based on the information available on the Lattes Platform. For statistical analysis, SPSS statistics and Microsoft Excel software were used. For the qualitative analysis, the word cloud visual resource was used. This paper contributes to the debate of the relation between postgraduation and tourism scientific production in Brazil.

Keywords: Graduation in Tourism; Teaching in Tourism; Knowledge Production; Brazil.

\section{Introdução}

A pós-graduação desempenha o importante papel de produção do conhecimento e inovação, visto que educação, ciência e tecnologia são pilares importantes para o desenvolvimento das nações.

Destaca-se que no Brasil o Sistema Nacional de Pós-graduação (SNPG) está em constante evolução, de 1998 a 2017, o número de cursos no país teve um aumento de $214 \%$.

Os cursos de mestrado e doutorado mais que dobraram em 15 anos, em 2000 haviam 1.439 programas disponíveis e em 2015 o número saltou para 3.905 (Capes, 2017).

Aponta-se que a década de 2000 foi marcada pela expansão das instituições federais de ensino superior, registrando um aumento considerável no número de cursos de graduação e pós-graduação no Brasil.

\footnotetext{
* Universidade de São Paulo (Brasil); E-mail: fausi@usp.br; https://orcid.org/0000-0002-3267-6377

** Universidade de São Paulo (Brasil); E-mail: mariana_lopes@usp.br; https://orcid.org/0000-0002-4526-6326

*** Universidade de São Paulo (Brasil); E-mail: mariana_tomazin@usp.br; https://orcid.org/0000-0003-0765-5010
} 
$\mathrm{Na}$ área do Turismo, foco da discussão da presente pesquisa, o aumento da produção bibliográfica especializada, a implementação dos cursos em nível de pós-graduação, a veiculação dos periódicos e eventos, assim como a criação da Associação Nacional de Pesquisadores de Turismo (ANPTUR) se deram também nessa década.

Posto isso, o objetivo principal desta investigação delineia-se em desvelar o universo da pós-graduação stricto sensu em Turismo no Brasil, primeiro a partir dos cursos oferecidos atualmente e na sequência, acerca dos docentes do universo escolhido, partindo da caracterização na dimensão individual destes profissionais para a construção do panorama coletivo dos programas investigados. Para isso, a pesquisa foi pautada em uma abordagem mista, contemplando dimensões qualitativas e quantitativas, de maneira complementar.

O universo total da pesquisa contemplou 10 programas stricto sensu de pós-graduação em Turismo e áreas afins, dentre instituições públicas e privadas do território brasileiro, sendo 08 mestrados acadêmicos, 02 mestrados profissionais e 05 programas contam também com o doutorado. A coleta de dados foi feita no universo, não em uma amostra, totalizando 131 docentes investigados.

Para tanto, o artigo está dividido da seguinte forma, inicialmente, apresenta-se o referencial teórico dividido em duas seções, a Pós-Graduação em Turismo no Brasil e Produção do Conhecimento do Turismo no Brasil, na sequência apresentam-se os procedimentos metodológicos, os resultados encontrados e por fim, as considerações finais.

\section{A pós-graduação em turismo no Brasil}

No Brasil os primeiros passos da pós-graduação foram dados a partir de 1930, sendo utilizado o termo "pós-graduação" pela primeira vez em 1940 no Artigo n 71 do Estatuto da Universidade do Brasil. Contudo, a implantação aconteceu definitivamente apenas em 1965 com o Parecer n 977/65 de 03 de dezembro de 1965 do Conselho Federal de Educação, conhecido como Parecer Sucupira ${ }^{1}$ (Velloso, 2014).

O referido documento apresenta a origem da pós-graduação em outros países, como a constituição da pós-graduação estadunidense, que por sua vez, é oriunda da influência germânica. Destaca-se também as transformações vividas nas universidades, que deixaram de ser apenas uma instituição ensinante e passarram a dedicar-se às atividades de pesquisa científica e tecnológica (Brasil, 1965).

No tópico sobre a necessidade da pós-graduação no Parecer Sucupira consta que em virtude da expansão da indústria brasileira era necessário aumentar o número crescente de profissionais criadores, capazes de desenvolver novas técnicas e processos, cuja formação precisava ir além da simples graduação (Brasil, 1965).

O Aviso Ministerial, ao solicitar a regulamentação, aponta, em síntese, os três motivos fundamentais que exigem, de imediato, a instauração de sistema de cursos pós-graduados: 1) formar professorado competente que possa atender à expansão quantitativa do nosso ensino superior garantindo, ao mesmo tempo, a elevação dos atuais níveis de qualidade; 2) estimular o desenvolvimento da pesquisa científica por meio da preparação adequada de pesquisadores; 3) assegurar o treinamento eficaz de técnicos e trabalhadores intelectuais do mais alto padrão para fazer face às necessidades do desenvolvimento nacional em todos os setores (Brasil, 1965: 02).

Nesse sentido, destaca-se o papel relevante instituído pela criação e regulamentação da pós-graduação no país, de superar as lacunas que até o presente momento ocorriam no âmbito da graduação e por ofertas estrangeiras, considerando a relevância da produção do conhecimento e da inovação em instituições de ensino superior.

Importante destacar a distinção entre a formação lato sensu e stricto sensu, que envolvem o universo da pós-graduação no Brasil:

As pós-graduações lato sensu compreendem programas de especialização incluem os cursos designados como MBA (Master Business Administration). Com duração mínima de 360 horas, ao final do curso o aluno obterá certificado e não diploma. Ademais são abertos a candidatos diplomados em cursos superiores e que atendam às exigências das instituições de ensino - Art. 44, III, Lei nº 9.394/1996 (Brasil, 2019).

As pós-graduações stricto sensu compreendem programas de mestrado e doutorado abertos a candidatos diplomados em cursos superiores de graduação e que atendam às exigências das instituições de ensino e ao edital de seleção dos alunos (Art. 44, III, Lei n ${ }^{\circ}$ 9.394/1996). Ao final do curso o aluno obterá diploma (Brasil, 2019).

Atualmente, a autorização, o reconhecimento e renovação de reconhecimento da pós-graduação stricto sensu em todos os estados brasileiros são obtidos a partir dos resultados da avaliação e do 
acompanhamento conduzidos pela Coordenação de Aperfeiçoamento de Pessoal de Nível Superior ${ }^{2}$ (CAPES), de acordo com as exigências previstas na legislação - Resolução CNE/CES n ${ }^{\circ}$ 1/2001, alterada pela Resolução CNE/CES n 24/2002 (CAPES, 2019).

Além do mestrado e doutorado acadêmico tem-se também a modalidade de mestrado profissional ${ }^{3}$ como curso de pós-graduação stricto sensu, o qual é definido pela CAPES como sendo: "modalidade voltada para a capacitação de profissionais, nas diversas áreas do conhecimento, mediante o estudo de técnicas, processos, ou temáticas que atendam a alguma demanda do mercado de trabalho" (Brasil, 2019).

$\mathrm{Na}$ CAPES os cursos de pós-graduação na área de Turismo estão vinculados à Coordenação de Administração Pública e de Empresas, Ciências Contábeis e Turismo, área de Ciências Sociais. Já no Conselho Nacional de Desenvolvimento Científico e Tecnológico (CNPq), órgão ligado ao Ministério da Ciência, Tecnologia, Inovações e Comunicações para incentivo à pesquisa no Brasil, propostas de pesquisas em Turismo são avaliadas no Comitê de Assessoramento de Arquitetura, Demografia, Geografia, Turismo e Planejamento Urbano e Regional.

Atualmente, ao acessar os cursos avaliados e reconhecidos pela Plataforma Sucupira ${ }^{4}$, pertecentes a área de avaliação - Administração Pública e de Empresas, Ciências Contábeis e Turismo, têm-se o total de 196 programas, sendo 185 que levam o nome Administração e 11 que levam o nome Turismo (CAPES, 2020).

Dessa forma, estrutura-se o recorte do estudo deste artigo, contemplando 10 programas de pós-graduação da área de Turismo, apenas desconsiderando o programa Gestão de Alimentos \& Bebidas da Universidade Anhembi Morumbi por motivo de escolha dos pesquisadores em virtude das linhas de pesquisas.

$\mathrm{Na}$ tabela 01 apresentam-se informações sobre os programas que compreendem o universo selecionado da investigação.

\section{Tabela 1: Cursos de Pós-Graduação em Turismo e áreas correlatas investigados na pesquisa}

\begin{tabular}{|c|c|c|c|c|}
\hline Início & Instituição & Programa & $\begin{array}{c}\text { Área de } \\
\text { Concentração }\end{array}$ & Linhas de Pesquisa \\
\hline $\begin{array}{l}1997(\mathrm{ME}) \\
2013(\mathrm{DO})\end{array}$ & UNIVALI & $\begin{array}{l}\text { Turismo e } \\
\text { Hotelaria }\end{array}$ & $\begin{array}{l}\text { Planejamento e } \\
\text { Gestão do Turismo e } \\
\text { da Hotelaria }\end{array}$ & $\begin{array}{l}\text { 1) Planejamento do Destino Turístico } \\
\text { 2) Gestão das Empresas de Turismo }\end{array}$ \\
\hline $\begin{array}{l}2000(\mathrm{ME}) \\
2015(\mathrm{DO})\end{array}$ & UCS & $\begin{array}{l}\text { Turismo e } \\
\text { Hospitalidade }\end{array}$ & $\begin{array}{l}\text { Desenvolvimento } \\
\text { regional do turismo }\end{array}$ & $\begin{array}{l}\text { 1) Turismo, organizações e sustentabilidade } \\
\text { 2) Turismo, cultura e educação }\end{array}$ \\
\hline $\begin{array}{l}2002(\mathrm{ME}) \\
2015(\mathrm{DO})\end{array}$ & UAM/SP & Hospitalidade & Hospitalidade & $\begin{array}{l}\text { 1) Dimensões e Contextos da Hospitalidade } \\
\text { 2) Hospitalidade na Competitividade em } \\
\text { Serviços }\end{array}$ \\
\hline $\begin{array}{l}2008(\mathrm{ME}) \\
2014(\mathrm{DO})\end{array}$ & UFRN & Turismo & $\begin{array}{l}\text { Turismo, } \\
\text { Desenvolvimento e } \\
\text { Gestão }\end{array}$ & $\begin{array}{l}\text { 1) Gestão em Turismo } \\
\text { 2) Turismo e Desenvolvimento Regional }\end{array}$ \\
\hline 2013 (ME) & UFPR & Turismo & $\begin{array}{l}\text { Turismo e } \\
\text { Desenvolvimento }\end{array}$ & $\begin{array}{l}\text { 1) Turismo, Sociedade e Ambiente } \\
\text { 2) Organizações Turísticas Públicas e Privadas }\end{array}$ \\
\hline $\begin{array}{l}2014(\mathrm{ME}) \\
2019(\mathrm{DO})\end{array}$ & USP & Turismo & $\begin{array}{l}\text { Desenvolvimento do } \\
\text { Turismo }\end{array}$ & $\begin{array}{l}\text { 1) Conhecimento e Tendências } \\
\text { 2) Processos e Inovação }\end{array}$ \\
\hline $2015(\mathrm{ME})$ & $\mathrm{UFF}$ & Turismo & Turismo e Sociedade & $\begin{array}{l}\text { 1) Turismo, planejamento e gestão } \\
\text { 2) Turismo, Cultura e Ambiente }\end{array}$ \\
\hline 2012 (MP) & UECE & $\begin{array}{l}\text { Gestão de } \\
\text { Negócios } \\
\text { Turísticos }\end{array}$ & $\begin{array}{l}\text { Gestão dos Negócios } \\
\text { e dos Territórios } \\
\text { Turísticos }\end{array}$ & $\begin{array}{l}\text { 1) Política, estratégia e gestão dos negócios } \\
\text { turísticos } \\
\text { 2) Turismo, território e desenvolvimento local }\end{array}$ \\
\hline 2016 (MP) & IFS & Turismo & Gestão de Turismo & $\begin{array}{l}\text { 1) Gestão de Destinos Turísticos: Sistemas, } \\
\text { Processos e Inovação } \\
\text { 2) Gestão de Turismo de Base Comunitária }\end{array}$ \\
\hline 2017 (ME) & UFPE & $\begin{array}{l}\text { Hotelaria e } \\
\text { Turismo }\end{array}$ & Hotelaria e Turismo & $\begin{array}{l}\text { 1) Gestão de Empresas Hoteleiras e Turísticas } \\
\text { 2) Turismo, Cultura e Sociedade }\end{array}$ \\
\hline \multicolumn{5}{|c|}{ ME - Mestrado Acadêmico } \\
\hline \multicolumn{5}{|c|}{ MP - Mestrado Profissional } \\
\hline \multicolumn{5}{|c|}{ DO - Doutorado Acadêmico } \\
\hline
\end{tabular}

Fonte: (Elaboração própria a partir dos sites dos programas de pós-graduação e da CAPES, 2020). 
Destaca-se que no Brasil o Sistema de Avaliação da Pós-Graduação desenvolvido pela CAPES é dividido em dois processos, um relacionado à entrada - Avaliação das Propostas de Cursos Novos, e o outro relacionado à permanência - Avaliação Periódica dos Cursos de Pós-Graduação (CAPES, 2020).

Como parte do processo de permanência ocorre a Avaliação Quadrienal dos cursos de pós-graduação, seguindo quesitos e indicadores estabelecidos no Conselho Técnico Científico da Educação Superior (CTC-ES). Os resultados desta avaliação são expressos em notas, numa escala de 1 a 7 , e determinam a continuidade do funcionamento dos programas no período subsequente, conforme deliberado pelo Conselho Nacional de Educação (CNE/MEC) (CAPES, 2020).

A última avaliação contemplou os seguintes quesitos: proposta do programa, corpo docente, corpo discente, produção intelectual e inserção social, cada qual relacionado a itens específicos. Os resultados finais da Avaliação Quadrienal 2017 acerca dos programas contemplados nesta investigação apontam que apenas a UNIVALI alcançou nota 05; a UAM, UCS, UFRN e a USP alcançaram nota 04; e a UFF, UFPR, IFS, UECE e UFPE alcançaram nota 03 (CAPES, 2017).

Os desafios impostos para a pós-graduação no Brasil são muitos, os programas necessitam cada vez mais propagar e difundir suas pesquisas, as quais demandam tempo, investimento e recursos humanos. Como é sabido o processo de construção do conhecimento percorre muitos caminhos, desde a concepção de uma problemática de pesquisa até os seus resultados. Contudo, para a continuidade dos cursos é necessário responder com os critérios exigidos pela Avaliação Quadrienal mencionada.

Situar o lugar da pós-graduação neste contexto se afirma como tarefa necessária para pensar os caminhos futuros no âmbito dos estudos turísticos no Brasil, dessa forma, a seção seguinte delineia alguns transcursos acerca da produção do conhecimento para embasar reflexões sobre o tema.

\section{Produção do conhecimento do turismo no Brasil}

A abordagem científica do turismo é relativamente recente, com crescimento do volume de bibliografias especializadas notadamente a partir dos anos 2000, como observado por Holanda, Widmer, \& Leal (2014), ao mapearem os trabalhos que analisam a produção científica brasileira em turismo no percurso histórico de constituição da área por meio de pesquisas bibliográficas e documentais.

Mirian Rejowski, como apontado pela referida pesquisa, professora sênior da Escola de Comunicações e Artes da Universidade de São Paulo e Professora Titular da Universidade Anhembi Morumbi, foi a primeira percussora a investigar especificamente a produção do conhecimento do turismo no Brasil, com sua tese de doutoramento (1993) e de livre-docência (1997) voltadas a temática, dedicando-se, posteriormente, a vários projetos de pesquisas, orientações a nível de graduação e pós-graduação sobre o tema.

Em pesquisas realizadas pela mesma autora aponta-se que outros pesquisadores brasileiros têm se dedicado ao assunto, com especificidades e abordagens distintas, como Gomes (2001 e 2004), Bertuzzo (2004), Leal (2006), Galvão Filho e Huertas Calvente (2006), Alberton e Lopes (2007), Hocayen-da-Silva e Gândara (2007), Bastos (2008), Moraes (2008), entre outros (Rejowski, 2010).

No mesmo estudo a autora apresenta produções científicas da área do turismo no exterior, apontando, destarte, o trabalho de Jafari e Aaser (1988) como importantes referenciais que subsidiaram seus trabalhos aqui no Brasil em 1993 e também os trabalhos de Hall (1991) na Austrália (Rejowski, 2010).

Na década de 2000 tem-se os estudos de Boterill (2002) acerca da produção científica do turismo no Reino Unido e Irlanda. E os estudos em Portugal e na Espanha se consolidando também nessa década. Assim como na Espanha, em 1996, os estudos turísticos passaram a constituir o sistema universitário. Com relação ao contexto sul-americano a autora aponta que não foram identificados estudos oriundos dessa região publicados até 2008 (Rejowski, 2010).

A partir de estudos feito por Santos \& Rejowski (2013: 158), ao abordarem um conjunto específico de periódicos científicos brasileiros contemplando o recorte temporal de 1990 a 2012, os autores obervaram que essa década também foi marcante, pois "foi a partir de 2003 que o número de artigos publicados [no Brasil] passou definitivamente a crescer em ritmo acelerado, movimento propiciado pelo surgimento de novos periódicos".

Nesse sentido, Holanda, Widmer, \& Leal (2014) ao mapearem os trabalhos que analisam a produção científica brasileira constatataram que até 2004 , existiam poucos periódicos de turismo e com a adoção do formato eletrônico aumentou o número de revistas específicas.

Não obstante, o periódico cumpre com a importante função de ser o principal meio formal de comunicação científica, fonte de informação escrita e pré-avaliada em uma determinada área ou campo de conhecimento, sendo editado periodicamente, a partir de uma política editorial definida (Santos \& Rejowski, 2013). 
Destaca-se que no Brasil utiliza-se a ferramenta Qualis-Periódico para classificar a produção científica dos programas de pós-graduação no que se refere aos artigos publicados em periódicos científicos. A classificação desta ferramente é feita pelos comitês de consultores de cada área da CAPES. Em virtude da nova metodologia adotada no Qualis-Periódicos no ano de 2019, o enquadramento dos estratos indicativos de qualidade se dão da seguinte forma: A1, o mais elevado, seguido pela ordem A2, A3, A4, B1, B2, B3, B4, e C, com peso zero (CAPES, 2019).

No âmbito da área Administração Pública e de Empresas, Ciências Contábeis e Turismo da CAPES é possível identificar muitos periódicos que debatem especificamente o desenvolvimento científico do turismo e áreas afins em diversas linhas de pesquisas, assim como, dialogam interdisciplinarmente com outras áreas, tanto periódicos nacionais, assim como internacionais.

A saber, alguns períodicos científicos são vinculados aos programas de pós-graduação brasileiros analisados por esta investigação. A Revista Turismo - Visão e Ação é vinculada à UNIVALI, a Revista Hospitalidade à UAM/SP, Caderno Virtual do Turismo à UFF, a Revista Rosa dos Ventos - Turismo e Hospitalidade à UCS, a Revista Turismo em Análise conta com o apoio do programa da USP, a Revista Turismo e Sociedade à UFPR, a Revista de Turismo Contemporâneo à UFRN.

Importante destacar que a Revista Turismo em Análise (RTA) foi pioneira como publicação científica específica de Turismo editada no Brasil pelo Departamento de Relações Públicas, Propaganda e Turismo da Escola de Comunicações e Artes da Universidade de São Paulo (ECA-USP) desde 1990.

A saber, o pioneirismo deste departamento da ECA-USP se deu também com a primeira oferta do curso de graduação em Turismo em uma instituição brasileira de ensino público com reconhecimento internacional, tendo o maior foco na formação acadêmica nas ciências sociais e humanas, no ano de 1973 (Sogayar \& Rejowki, 2011). E no âmbito da pós-graduação stricto sensu, foi também neste departamento da ECA-USP, que ofereceu-se o primeiro curso de mestrado em Comunicação, com uma linha em Lazer e Turismo, no ano de 1993, o qual teve sua data de encerramento por volta de 2005 e 2008 (Freitas, 2018).

Ressalta-se que a Revista Brasileira de Pesquisa em Turismo é vinculada à Associação Nacional de Pesquisadores de Turismo (ANPTUR), que reúne instituições brasileiras de programas de pós-graduação em Turismo, Hotelaria, Lazer e áreas afins, fundada desde 2002.

Todo ano a ANPTUR realiza um seminário que já vai para sua décima sétima edição em 2020, que assim como os periódicos, também cumpre com um importante papel, o de consolidar os eventos científicos como espaços de produção e transposição do conhecimento entre pesquisadores de diversas instituições de ensino. Destaca-se, inclusive, que no ano de 2008 criou-se um grupo de trabalho no referido evento intitulado como "Produção científica em Turismo e Hospitalidade".

O amadurecimento da Associação Nacional de Pesquisa e Pós-Graduação em Turismo (ANPTUR), que começou a promover, em 2005 , um seminário anual que congrega os principais pesquisadores do turismo nacional bem como professores e pesquisadores de todas as regiões do país, foi um marco para a consolidação da pesquisa em turismo no Brasil. Em 2007, a ANPTUR deu início à publicação da Revista Brasileira de Pesquisa em Turismo, um importante veículo para a disseminação do conhecimento científico na área. Assim, através destas iniciativas, pesquisadores dos Programas de Turismo e áreas correlatas têm dois excelentes canais de comunicação com a comunidade acadêmica de turismo nacional (Leal, 2011: 145).

Outra importante base de dados específica na área do Turismo é o site Publicações de Turismo, criado em 2011 e desenvolvido pelo Professor Glauber Santos. Atualmente, é um projeto de extensão do Programa de Pós-Graduação em Turismo da Escola de Artes, Ciências e Humanidades da USP (EACH-USP). O banco de dados é composto por informações dos artigos publicados em periódicos científicos iberoamericanos de Turismo que utilizam o sistema Open Journal Systems (OJS). As informações são coletadas diretamente dos metadados fornecidos pelas revistas, constando atualmente 37 periódicos indexados (Publicações de Turismo, 2019).

Não obstante, outra contribuição importante relacionada à sistematização da produção do conhecimento no país é a construção de um Tesauro Brasileiro de Turismo sob coordenação de Rejowski (Rejowski \& Barbanti, 2019; Rejowski \& Kobashi, 2011)

O Tesauro Brasileiro de Turismo é uma linguagem documental que representa de forma normalizada os conceitos do campo do Turismo mediante um conjunto de termos que se manifestam em estruturas lógico-semânticas, a ser utilizado como base de conhecimento, catalogação/indexação e suporte para pesquisa online (Rejowski \& Barbanti, 2019: 190). 
Após várias etapas de pesquisas, consulta a modelos de tesauros, sistematização, construções metodológicas, adequações de softwares, validação com experts da área e demais percursos construídos ao longo de anos, o Tesauro Brasileiro de Turismo foi disponibilizado à comunidade científica como um instrumento de apoio às pesquisas turísticas, estruturado em 17 categorias temáticas, que a saber são:

1) alimentos e bebidas; 2) ciência e informação em turismo; 3) comunicação turística; 4) economia e turismo;

5) educação e formação em turismo; 6) eventos turísticos; 7) hospitalidade no turismo; 8) lazer e turismo;

9) legislação turística; 10) organismos de turismo; 11) patrimônio turístico; 12) planejamento turístico;

13) política de turismo; 14) serviços turís- ticos; 15) sociedade e turismo; 16) tecnologia da informação e comunicação em turismo; e 17) transportes no turismo (Rejowski \& Barbanti, 2019: 191).

Nesse sentido, o panorama apresentado demonstra como tem se consolidado a construção do conhecimento na área, especificamente no caso brasileiro, onde notadamente a partir da década de 2000 tem-se o surgimento de novos periódicos, sobretudo, onlines, e a possível relação direta com o fortalecimento de pesquisas no âmbito da pós-graduação.

\section{Metodologia}

Para alcançar o objetivo de desvelar aspectos da pós-graduação stricto sensu em Turismo no Brasil o presente trabalho estruturou-se a partir de dois objetivos específicos, o de reconhecer a oferta atual dos cursos de pós-graduação da área e na sequência o de identificar o perfil dos docentes vinculados ao universo escolhido, compreendendo as respectivas dimensões individuais destes profissionais e assim caracterizando os programas que pertecem, subsidiando assim uma compreensão do panorama institucional dos programas. Para isso, a pesquisa adotou uma abordagem mista, contemplando as dimensões qualitativa e quantitativa.

A abordagem qualitativa realizada é classificada como exploratória e descritiva, a qual consistiu de revisão bibliográfica, por meio de bases de dados científicos, sobre o tema da produção científica em turismo e acerca do percurso histórico da pós-graduação.

Realizou-se também consultas aos sites dos órgãos que atuam diretamente com a expansão, consolidação e incentivo da pós-graduação stricto sensu no Brasil - CAPES e CNPq, utilizando as ferramentas digitais disponíveis da plataforma Sucupira, do Qualis-Periódicos e da plataforma Lattes. Os sites dos programas de pós-graduação e dos periódicos da área também foram consultados.

A síntese da pesquisa qualitativa foi apresentada nas seções iniciais deste artigo, reconhecendo o quadro atual dos programas de pós-graduação stricto sensu em Turismo vinculados à CAPES, que se estabelecem na totalidade de 11 programas, sendo 10 programas contemplados para a análise desta investigação, conforme apresentado na Tabela 1.

Acerca do objetivo específico de identificar o perfil dos docentes dos programas brasileiros de pós-graduação stricto sensu em Turismo optou-se por uma abordagem quantitativa. O universo dos 10 programas de pós-graduação constitui-se por 15 cursos, sendo 05 doutorados, 08 mestrados acadêmicos, 02 mestrados profissionais, em um universo tanto de instituições públicas como privadas.

A postura metodológica nesta etapa criou uma varredura de dados, ou seja, ao invés de amostragem, utilizou-se todo o universo de professores $(\mathrm{u}=131)$ cadastrados nos programas investigados, totalizando em 131 docentes vinculados.

A ferramenta utilizada foi o currículo Lattes, um sistema criado pelo CNPq, que integra informações sobre bases de dados de currículos, grupos de pesquisa, adotado pela maioria das instituições de fomento, universidades e institutos de pesquisas do Brasil, e é atualmente, o padrão nacional de currículo de estudantes e pesquisadores do país (CNPq, 2020).

A coleta na ferramenta ocorreu durante a segunda quinzena do mês de abril de 2020. Ressalta-se ainda que há disponibilizado no site da Capes um banco de dados sobre os profissionais das Instituções Públicas de Ensino Superior, entretanto, optou-se por não utilizar essa fonte haja vista sua atualização e também, por que a estratégia da busca direta nos currículos individuais permitiu maior detalhe na caracterização do perfil dos docentes investigados.

A investigação de dados teve duas etapas distintas: a primeira consistiu em pesquisa de gabinete na qual as informações levantadas foram as seguintes: instituição de atuação; sexo dos profissionais; $1^{\circ}$ graduação; $2^{\circ}$ graduação; $1^{\circ}$ mestrado; $2^{\circ}$ mestrado; $1^{\circ}$ doutorado; $2^{\circ}$ doutorado; pós doutorado; doutorado sanduíche; principais trabalhos publicados; áreas de interesse; áreas de concentração dos programas. 
A decisão de coleta dessas informações, por sua vez, não aconteceu de maneira randômica. Todas essas estão atreladas fortemente com o objetivo principal de construir um quadro panorâmico do perfil dos docentes de pós-graduações em Turismo no território brasileiro, tanto em escala individual - com tratamento de dados voltado para o profissional - como em escala coletiva - com análises apresentadas por programa.

Cabe ainda alguns apontamentos relevantes em relação as escolhas dos dados coletados: identificou-se que alguns professores possuíam mais de uma graduação e/ou pós-graduação. Por esse motivo, decidiu-se coletar ambas em graduação, mestrado e doutorado. Para o pós-doutorado, ao invés de uma resposta numérica (quantos?), escolheu-se trabalhar com nominal (sim ou não); por fim, em relação aos principais trabalhos, o critério utilizado foi o de coletar as cinco publicações definidas pelos cientistas como mais relevantes em suas carreiras por meio da ferramenta oferecida pelo próprio Currículo Lattes (o uso da estrela amarela), podendo estas serem livros, artigos em periódicos, trabalhos apresentados em congresso etc.

A decisão de coleta de dados por meio de varreduras apresenta pontos positivos, pois há maior consistência dos dados, levando em consideração que todo o universo foi investigado e posto em análise neste trabalho. Dessa maneira, não há projeções nem conjecturas estatísticas, mas dados absolutos no que se propôs investigar.

Contudo, coletar dados por meio de uma plataforma que precisa ser regularmente atualizada e que depende do preenchimento individual pode ter contribuído para elaboração de informações desatualizadas e a possibilidade de impacto no universo, para mais ou para menos.

No segundo momento, ocorreu a análise de dados. Para as análises estatísticas utilizou-se o software SPSS statistics e o Microsoft Excel. Por se tratar da análise de um universo (e não de uma amostra), tratou-se os dados em frequências, porcentagens e tabelas. Houve também o cruzamento de dados das instituições e formações dos profissionais. Os resultados e análises estão dispostos na próxima seção.

\section{Resultados}

O universo de pesquisa da presente pesquisa constitui-se no total de 131 docentes de 10 Programas de Pós-Graduação em Turismo no território brasileiro, distribuídos pelas instituições como apresentado pela Tabela 2.

Tabela 2: Número de docentes por instituição

\begin{tabular}{|c|c|c|c|}
\hline Instituição & Frequência & Porcentagem & Porcentagem Válida \\
\hline UFPR & 16 & 12,2 & 12,2 \\
\hline USP & 16 & 12,2 & 12,2 \\
\hline UFRN & 16 & 12,2 & 12,2 \\
\hline UCS & 15 & 11,5 & 11,5 \\
\hline UFF & 13 & 9,9 & 9,9 \\
\hline UNIVALI & 13 & 9,9 & 9,9 \\
\hline UFPE & 13 & 9,9 & 9,9 \\
\hline UECE & 12 & 9,2 & 9,2 \\
\hline UAM & 09 & 6,9 & 6,9 \\
\hline IFS & 08 & 6,1 & 6,1 \\
\hline TOTAL & 131 & 100 & 100 \\
\hline
\end{tabular}

Fonte: Elaboração própria a partir dos dados coletados.

Os programas de pós-graduação da UFPR, USP e UFRN aparecem com a maior número de docentes, 16 cada, a saber, os programas evidenciados possuem cursos de mestrado acadêmico e doutorado. Enquanto o IFS aparece com menor frequência, tendo 8 professores afiliados ao programa e possuindo o curso de mestrado profissional, que iniciou em 2016. 
No que diz respeito ao sexo, os resultados encontrados entre masculino e feminino são equilibrados, com porcentagem de $51,1 \%$ para masculino e $48,9 \%$ para o feminino.

\section{Tabela 3: Sexo dos docentes}

\begin{tabular}{|c|c|c|c|}
\hline & Frequência & Porcentagem & Porcentagem Válida \\
\hline Masculino & 67 & 51,1 & 51,1 \\
\hline Feminino & 64 & 48,9 & 48,9 \\
\hline TOTAL & 131 & 100,0 & 100,0 \\
\hline
\end{tabular}

Fonte: Elaboração própria a partir dos dados coletados.

No que se refere à graduação dos professores (primeira e segunda), foram encontradas 149 formações distribuídas em 43 cursos diferentes. Esse resultado superior ao universo pesquisado (131) indica que 18 docentes possuem uma segunda formação.

A maior frequência de pesquisadores tem formação em Turismo ( $\mathrm{n}=42$ ou $28,2 \%$ do universo). Há também formações similares, como o caso de Turismo e Hotelaria ( $\mathrm{n}=2$ ou 1,3\%) e Gestão em Hotelaria, Turismo e Lazer ( $n=2$ ou 1,3\%). Administração $(n=14$ ou 9,4\%) e Geografia ( $n=12$ ou 8,1\%) aparecem em números absolutos, respectivamente, como a segunda e terceira colocação. Um achado curioso está para algumas formações que podem ser consideradas não tão convencionais, como o caso de Enfermagem e Engenharia Elétrica (ambos com n=1 ou 0,7\%).

Mediante ao grande número de graduações e pós-graduações encontrados, buscou-se adotar um critério para otimizar a organização e apresentação dos dados na análise, adotando então, a estratégia para agrupar esses dados sem prejudicar as análises que seguem doravante pelo critério de classificação adotado pela CAPES.

A referida classificação constitui-se por 49 áreas de avaliação, agrupadas por critério de afinidades em dois níveis: Colégios e Grandes Áreas, na qual o segundo nível está inserido no primeiro. São 03 Colégios, sendo eles: 1) Colégio de Humanidades; 2) Colégio de Ciências da Vida; e 3) Colégio de Ciências Exatas, Tecnológicas e Multidisciplinar. A Tabela 4 indica algumas das grandes áreas incluídas dentro de cada Colégio.

Tabela 4: Colégios e grandes áreas estabelecidos pela CAPES

\begin{tabular}{|c|c|c|}
\hline Colégio De Humanidades & Colégio de Ciências da Vida & $\begin{array}{c}\text { Colégio de Ciências } \\
\text { Exatas, Tecnológicas e } \\
\text { Multidisciplinar }\end{array}$ \\
\hline Antropologia/ Arqueologia & Educação Física & Geociências \\
\hline Sociologia/ Filosofia & Nutrição & Química \\
\hline Economia & Enfermagem & Engenharias \\
\hline $\begin{array}{c}\text { Administração / Administração } \\
\text { de empresas }\end{array}$ & Farmácia & Biotecnologia \\
\hline Turismo & Zootecnia & Ciência da Computação Ambientais \\
\hline Ciências contábeis & Ciências Agrárias & Ciências da Computação \\
\hline Economia & Ciências Biológicas & \\
\hline
\end{tabular}

Fonte: Elaboração própria a partir dos dados coletados da CAPES.

Ainda que a classificação dos Colégios seja instituída pela CAPES para as áreas de pós-graduação (excluindo a graduação), optou-se, neste trabalho, por adotar um critério único para agrupar e analisar todas as formações. Isso ocorre para dar coerência analítica e reforçar um rigor metodológico, evitando assim critérios múltiplos e uma confusão nas análises do que foi coletado. Dessa forma, a Tabela 5 aponta o cruzamento das graduações dos docentes (já agrupadas pelos Colégios CAPES) pelas instituições de atuação da pós-graduação do universo investigado. 
Tabela 5: Graduação dos docentes de pós-graduação em Turismo e áreas afins:

\begin{tabular}{|c|c|c|c|c|c|c|}
\hline \multirow[t]{2}{*}{ Instituições } & \multicolumn{2}{|c|}{ Humanidades } & \multicolumn{2}{|c|}{ Ciências da Vida } & \multicolumn{2}{|c|}{$\begin{array}{c}\text { Ciências Exatas e } \\
\text { Tecnológicas }\end{array}$} \\
\hline & Sim & Não & Sim & Não & Sim & Não \\
\hline UFPR & 16 & 0 & 0 & 16 & 0 & 16 \\
\hline USP & 12 & 4 & 3 & 13 & 2 & 14 \\
\hline UECE & 11 & 1 & 1 & 11 & 0 & 12 \\
\hline UFF & 12 & 1 & 1 & 12 & 0 & 13 \\
\hline UNIVALI & 12 & 1 & 1 & 12 & 2 & 11 \\
\hline UFPE & 13 & 0 & 0 & 13 & 0 & 13 \\
\hline UAM & 8 & 1 & 0 & 9 & 1 & 8 \\
\hline UFRN & 12 & 3 & 1 & 14 & 2 & 13 \\
\hline IFS & 7 & 1 & 2 & 6 & 0 & 8 \\
\hline UCS & 13 & 2 & 2 & 13 & 1 & 14 \\
\hline
\end{tabular}

Fonte: Elaboração própria a partir dos dados coletados.

O resultado encontrado demonstra que dentro do Colégio de Humanidades há destaque para UFPR e UFPE, na qual ambas apresentam 100\% dos professores vinculados com formação em humanas. Outra observação relevante é que dentro do panorama geral dos Colégios utilizados para analisar a formação desses profissionais, pode-se notar um perfil majoritário dentro das áreas humanas.

Dentro das Ciências da Vida, a USP, a UCS e e IFS se sobressaem. Entre algumas das graduações contidas nesse colégio foram encontradas: Educação física (n=3), Enfermagem $(n=1)$ e Zootecnia $(n=1)$. Já nas Ciências Exatas e Tecnológicas a USP, a UFRN e a UNIVALI apresentam um número maior de profissionais, com destaque para as áreas da engenharia.

No âmbito das formações a nível de mestrado foram encontradas 60 formações distintas, com destaque para a formação em Administração, que representa 18\% dos investigados $(n=25)$. Geografia aparece na segunda colocação, com 17 profissionais (12\%) e Ciências da Comunicação e Turismo e Hotelaria em terceiro com 7 docentes cada um (5\%). Dentro do universo, 9 docentes possuem dois mestrados (cerca de 7\%) e 3 deles não informam os mestrados no currículo. A Tabela 6 apresenta a sistematização das formações a nível de mestrado classificadas dentro dos Colégios da Capes.

Tabela 6: Mestrado dos docentes de pós-graduação em Turismo e áreas afins:

\begin{tabular}{|c|c|c|c|c|c|c|}
\hline \multirow[t]{2}{*}{ Instituições } & \multicolumn{2}{|c|}{ Humanidades } & \multicolumn{2}{|c|}{ Ciências da Vida } & \multicolumn{2}{|c|}{$\begin{array}{c}\text { Ciências Exatas e } \\
\text { Tecnológicas }\end{array}$} \\
\hline & Sim & Não & Sim & Não & Sim & Não \\
\hline UFPR & 16 & 0 & 0 & 16 & 0 & 16 \\
\hline USP & 13 & 2 & 2 & 13 & 0 & 15 \\
\hline UECE & 10 & 0 & 0 & 10 & 1 & 9 \\
\hline UFF & 12 & 1 & 0 & 13 & 1 & 12 \\
\hline UNIVALI & 9 & 4 & 1 & 12 & 3 & 10 \\
\hline UFPE & 12 & 1 & 1 & 12 & 2 & 11 \\
\hline UAM & 9 & 0 & 0 & 9 & 0 & 9 \\
\hline UFRN & 12 & 4 & 1 & 15 & 3 & 13 \\
\hline IFS & 8 & 0 & 0 & 8 & 0 & 8 \\
\hline UCS & 11 & 4 & 1 & 14 & 3 & 12 \\
\hline
\end{tabular}

Fonte: Elaboração própria a partir dos dados coletados.

Já no âmbito a nível de doutorado se identificaram 57 diferentes formações. Essa informação estava disponível na totalidade do universo investigado, inclusive, se destaca que cinco docentes possuem 
dois títulos de doutor, totalizando 136 títulos analisados. Dessa vez, o doutoramento em Geografia surge com a maior frequência ( $n=27$ ou 20\%), seguido de Administração ( $n=20$ ou 14,7\%) e Ciências da Comunicação ( $\mathrm{n}=9$ ou 6,6\%). A formação em Turismo, Turismo e Hotelaria e Administração e Turismo somam 10 docentes, com frequências respectivas: 1, 4 e 5. A Tabela 7 apresenta a sistematização das formações a nível de doutorado classificadas dentro dos Colégios da Capes.

Tabela 7: Doutorado dos docentes de pós-graduação em Turismo e áreas afins:

\begin{tabular}{|c|c|c|c|c|c|c|}
\hline \multirow{2}{*}{ Instituições } & \multicolumn{2}{|c|}{ Humanidades } & \multicolumn{2}{c|}{ Ciências da Vida } & \multicolumn{2}{c|}{$\begin{array}{c}\text { Ciências Exatas e } \\
\text { Tecnológicas }\end{array}$} \\
\hline & Sim & Não & Sim & Não & Sim & Não \\
\hline UFPR & 16 & 0 & 0 & 16 & 0 & 16 \\
\hline USP & 14 & 2 & 1 & 15 & 1 & 15 \\
\hline UECE & 11 & 1 & 0 & 12 & 1 & 11 \\
\hline UFF & 13 & 0 & 0 & 13 & 0 & 13 \\
\hline UNIVALI & 8 & 5 & 0 & 13 & 5 & 8 \\
\hline UFPE & 13 & 0 & 0 & 13 & 0 & 13 \\
\hline UAM & 9 & 0 & 0 & 9 & 0 & 9 \\
\hline UFRN & 12 & 4 & 1 & 15 & 3 & 13 \\
\hline IFS & 7 & 1 & 0 & 8 & 1 & 7 \\
\hline UCS & 12 & 3 & 1 & 14 & 2 & 13 \\
\hline
\end{tabular}

Fonte: Elaboração própria a partir dos dados coletados.

Com o intuito de sintetizar as informações apresentadas a partir das tabelas dos Colégios CAPES (graduação, mestrado e doutorado), elaborou-se um sistema de pontuação (score) para cada instituição. Para isso, somou-se a quantidade de docentes de cada Colégio, levando em consideração todas as titulações. Ao fim, calculou-se, individualmente, a porcentagem apresentada por cada Instituição para cada colégio. Esse cálculo da porcentagem precisou ser realizado individualmente devido as diferentes quantidades de profissionais em cada programa e é apresentado na Tabela 8.

Tabela 8: Síntese das formações (graduação/mestrado/ doutorado) dos docentes investigados:

\begin{tabular}{|c|c|c|c|}
\hline \multirow{2}{*}{ Instituição } & \multicolumn{3}{|c|}{ Colégios } \\
\cline { 2 - 4 } & Humanidades & Saúde & Exatas \\
\hline UFPR & $100 \%$ & $0 \%$ & $6 \%$ \\
\hline USP & $81,5 \%$ & $12,5 \%$ & $6 \%$ \\
\hline UECE & $91 \%$ & $3 \%$ & $2,6 \%$ \\
\hline UFF & $94,8 \%$ & $2,6 \%$ & $25 \%$ \\
\hline UNIVALI & $70 \%$ & $5 \%$ & $5 \%$ \\
\hline UFPE & $92.5 \%$ & $2.5 \%$ & $3,7 \%$ \\
\hline UAM & $96,3 \%$ & $0 \%$ & $17 \%$ \\
\hline UFRN & $76,5 \%$ & $6,5 \%$ & $4 \%$ \\
\hline IFS & $88 \%$ & $8 \%$ & $13 \%$ \\
\hline UCS & $78,3 \%$ & $8,7 \%$ & \\
\hline
\end{tabular}

Fonte: Elaboração própria a partir dos dados coletados. 
De maneira geral, há uma homogeneidade considerável na análise acima, na qual o maior índice de formação está dentro do campo das humanidades em todos os programas. Destaca-se a UFPR que possui todo corpo docente com formação no colégio de humanidades; a USP ganha destaque por ter o maior quantitativo de profissionais ligados à escola da saúde, com ênfase na formação de Educação Física (infere-se que essa relação aconteça porque os docentes estão vinculados ao curso da graduação em Lazer e Turismo); e a UNIVALI é o programa com maior porcentagem nas áreas exatas, com destaque para engenharias.

Os programas do IFS, UCS e UFRN também apresentam um corpo docente menos homogêneo, comparado ao restante dos programas. Pode-se deduzir que a grande quantidade de estudos qualitativos dentro da área do turismo está atrelada a essa homogeneidade de formação no colégio de humanidades.

Sobre a realização de Doutorado Sanduíche em instituições internacionais apenas 9,9\% de todo o universo dos docentes da pós-graduação o realizaram, sendo os principais países das instituições: Portugal, França e Espanha e também Estados Unidos.

Sobre pós-doutoramento, tem-se que 53 (40,5\%) professores possuem essa experiência. O pós-doutorado não é uma titulação, como acontece nos casos de mestrado e doutorado e, portanto, não há necessidade de defesa de uma dissertação ou tese. De acordo com o portal do Ciências sem Fronteiras (2020) a finalidade de um pós-doutorado é a de "capacitar e atualizar os conhecimentos de um pesquisador que ocorre por meio de um estágio e o desenvolvimento de um projeto técnico-científico que ocorre em até 12 meses".

No que se refere às publicações de artigos acadêmicos publicados pelos docentes dos programas, foram utilizados dois critérios investigativos, ambos por uma perspectiva temporal: o primeiro deles tratou de trabalhos publicados a partir de 2015, enquanto o segundo trabalhos publicados em sua totalidade.

As instituições IFS e UFF apresentam os menores números totais de trabalhos publicados pelo docentes e também de menor produção nos últimos cinco anos. Quanto ao maior número total de publicações, USP e UFRN e UCS ocupam as primeiras colocações. Entretanto, cabe uma observação pertinente: apesar da USP liderar o ranking de publicações totais, notou-se que nos últimos cinco anos as universidades UFRN e Univali tiveram números superiores de produção, com valores em 273 e 260, respectivamente contra 244 da USP.

No que diz respeito das publicações de livros, a USP surge, novamente, na primeira colocação totalizando 146 (centro e quarenta e seis) livros, seguido pela UCS com 93 (noventa e três) e pela UECE com 66 (sessenta e seis):

Também foram coletadas as principais publicações de cada pesquisador. Decidiu-se que o critério utilizado para estabelecer a quantidade de trabalhos de cada pesquisador seria de até cinco, identificados por meio da ferramenta "estrela", disponível pela própria plataforma Lattes. Esse recurso permite o pesquisador evidenciar suas cinco pesquisas mais relevantes (incluindo publicações em periódicos, livros, projetos de pesquisa etc.), portanto, esse número trata de uma amostra e não de um universo, como no caso das publicações totais e publicações de livros demonstradas acima.

Considerou-se esse critério metodologicamente coerente, pois cria um parâmetro quantitativo e ao mesmo tempo imbuído de pessoalidade, visto que a atribuição da "estrela" fica a critério de cada cientista. Por outro lado, esse critério apresenta dois pontos fracos: primeiramente, não leva em consideração todas as obras do universo; em segundo lugar, houve casos de docentes que não atribuiram "estrela" alguma para suas obras, reduzindo assim a amostragem pretendida. Caso todo o universo analisado atribuísse todas as cinco "estrelas" permitidas pelo Lattes, a amostra trabalhada seria de 655, contudo, houve 79 evasões, derrubando esse número para 576.

A análise que se fez em cima desse quantitativo de artigo foi a de identificar trabalhos nacionais e trabalhos internacionais por curso de pós-graduação. Identificar essa produção, mesmo que em termos relativos, ajuda a construir um diagnóstico importante para esses programas, visto a produção internacional/internacionalização do conhecimento ser uma ação importante para programas alcançarem notas maiores na Avaliação Quadrienal da Capes, que define a continuidade do funcionamento dos programas de pós-gradiação no Brasil no período subsequente, a partir das notas que variam entre 1 a 7 . Foram analisados 576 produções, a tabela 9 apresenta os resultados das produções sinalizadas pelos docentes como relevantes e classificadas em nacionais e internacionais. 
Tabela 9: Principais produções nacionais e internacionais dos programas

\begin{tabular}{|c|c|c|}
\hline Instituição & Trabalhos e Publicações Nacionais & Trabalhos e Publicações Internacionais \\
\hline UFRN & 50 & 24 \\
\hline USP & 47 & 24 \\
\hline UCS & 44 & 23 \\
\hline UFPE & 38 & 21 \\
\hline UFPR & 52 & 18 \\
\hline UNIVALI & 42 & 16 \\
\hline UFF & 39 & 16 \\
\hline UAM & 23 & 16 \\
\hline UECE & 35 & 15 \\
\hline IFS & 28 & 5 \\
\hline TOTAL & 398 & 178 \\
\hline
\end{tabular}

Fonte: Elaboração própria a partir dos dados coletados.

Como observado acima, a UFRN, a USP e a UCS apresentam os maiores índices de trabalhos internacionais identificados na amostra, representando cerca de $30 \%$ da produção de seus docentes. A UECE e o IFS apresentaram os menores números de publicações internacionais. Em números totais, é possível perceber que menos de $30 \%$ do que os pesquisadores consideram como mais relevantes em suas pesquisas o que está sendo publicado internacionalmente.

Outra análise importante que este artigo se propõe a fazer é em relação as áreas de interesse dos docentes de turismo. Para isso, coletou-se a descrição individual acerca de suas áreas de interesse em pesquisas pelo resumo disponível no Currículo Lattes. O resumo, por sua vez, é de livre criação, o que permite cada pesquisador preencher da maneira que achar conveniente. A estratégia escolhida para analisar esses dados qualitativos foi a nuvem de palavras (wordcloud). Esse é um recurso visual que identifica a frequência de palavras de um texto e atribui importância por meio do tamanho de fonte. Isso significa que quanto mais vezes uma palavra aparece em determinado texto, com mais destaque ela surge na nuvem de palavras, ao passo que, quanto menor a frequência das palavras, essas recebem menor destaque visual. Exclui-se do corpo de texto as preposições, conjunções e artigos (no, na, de, para, um, uma), para tornar a representação visual mais polida. A figura abaixo é a representação geral da área de interesse de todos os professores investigados:

Figura 1: Nuvem de palavras sobre os áreas de interesses em pesquisas dos docentes

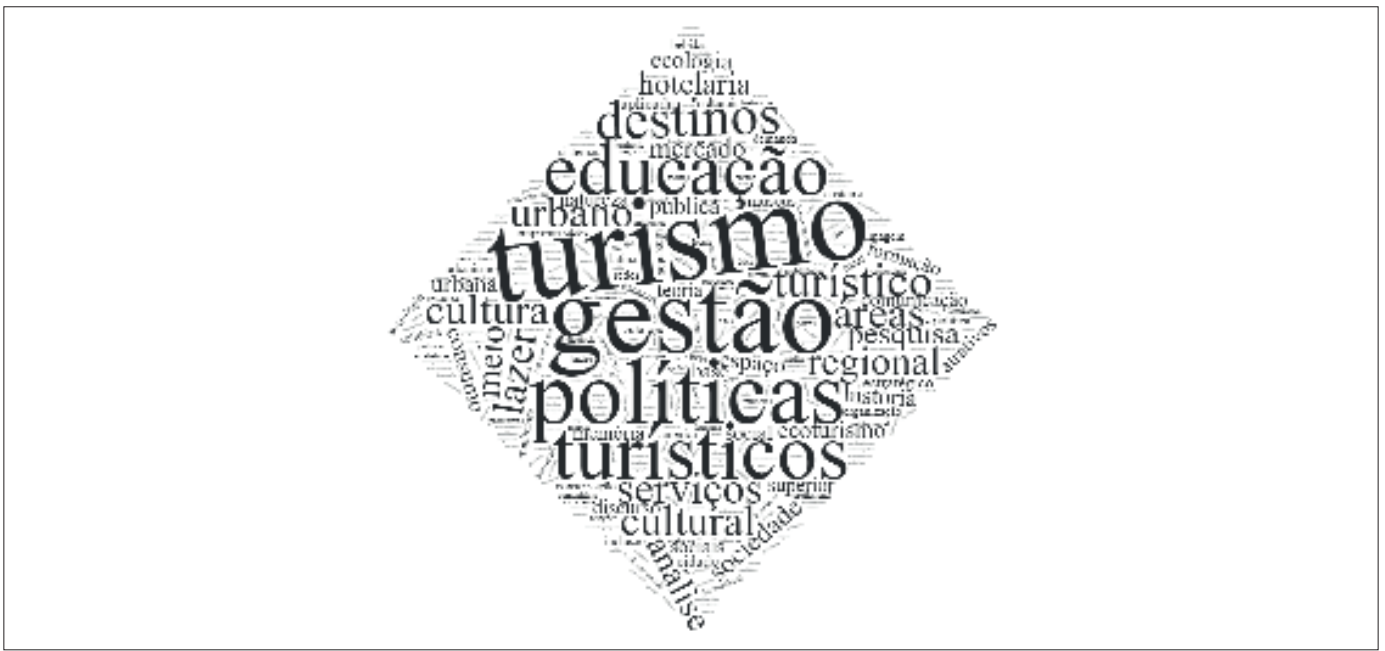

Fonte: Elaboração própria a partir dos dados coletados. 
Além da palavra turismo (que por lógica surge como o principal termo de interesse dos investigados), as palavras: gestão, destinos, educação, política, urbano, hotelaria, serviços, cultura e lazer surgem como destaque na nuvem de palavras. Entretanto, esse primeiro tratamento indica um aspecto geral dos dez programas investigados.

Neste sentido, propondo-se realizar uma análise mais aprofundada, os interesses foram particionados por instituições gerando mais dez novas nuvens de palavras. Levando em consideração a limitação de páginas de um artigo e também respeitando o aspecto visual, os cinco termos mais evidentes foram reorganizados e apresentados na tabela abaixo:

\section{Tabela 10: Áreas de interesses de pesquisa dos docentes por instituição}

\begin{tabular}{|c|c|}
\hline Instituição & Palavras em destaque na nuvem de palavras \\
\hline USP & Turismo; Educação; Lazer; Planejamento; Urbano \\
\hline UFRN & Turismo; Planejamento; Estratégia; Gestão; Destinos \\
\hline UFPR & Turismo; Planejamento; Desenvolvimento; Políticas; Gestão \\
\hline UFF & Turismo; Antropologia; Planejamento; Museus; Meio-ambiente \\
\hline UECE & Turismo; Economia; Educação; Ambiental; Gestão \\
\hline UCS & Turismo; Planejamento; Análise; Discurso; Gestão \\
\hline UNIVALI & Turismo; Gestão; Marketing; Gastronomia; Hospitalidade \\
\hline UFPE & Turismo; Gestão; Serviços; Comportamento; Consumidor \\
\hline UAM & Turismo; Hospitalidade; Serviços; Gastronomia; Empresarial \\
\hline IFS & Turismo; Acessibilidade; Territorial; Planejamento; Ambiental \\
\hline
\end{tabular}

Fonte: Elaboração própria a partir dos dados coletados.

Pode-se notar que instituições como Univali, UFPE e UAM possuem um corpo docente com interesses de pesquisas voltadas para o mercado e consumo, além da gastronomia. O IFS, por sua vez, indica ter pesquisadores ligados ao território e planejamento urbano enquanto a USP indica interesses ao Urbano, Turismo e Lazer; UCS, UFRN e UFPR parecem estar voltadas para produção de pesquisas ligadas ao Planejamento e Gestão com relação à atividade turística, visto que todas apresentam uma interseção nas palavras "turismo", "gestão" e "planejamento". Já a UECE parece estar mais concentrada no campo da gestão e economia do turismo, mas não no planejamento; e, a UFF, surge, aparentemente como a mais multidisciplinar das instituições.

Os resultados encontrados nas nuvens de palavras são apresentados na tabela 11 comparados com as áreas de concentração dos programas.

\section{Tabela 11: Nuvem de palavras de áreas de interesses de pesquisas e as áreas dos programas de pós-graduação}

\begin{tabular}{|c|c|c|}
\hline Instituição & Análise da nuvem de palavras & Áreas de concentração dos programas \\
\hline USP & Turismo, Lazer e Urbano & Desenvolvimento do Turismo \\
\hline UFRN & Planejamento e Gestão do turismo & Turismo, desenvolvimento e Gestão \\
\hline UFPR & Planejamento e Gestão do turismo & Turismo e desenvolvimento \\
\hline UCS & Planejamento e Gestão do turismo & Desenvolvimento Regional do Turismo \\
\hline UECE & Gestão do Turismo & Gestão dos Negócios e dos Territórios Turísticos \\
\hline UNIVALI & Mercado do turismo e consumo & Planejamento e Gestão do Turismo e Hotelaria \\
\hline UFPE & Mercado do turismo e consumo & Hotelaria e Turismo \\
\hline UAM & Mercado do turismo e consumo & Hospitalidade \\
\hline IFS & Planejamento e Território & Gestão do Turismo \\
\hline UFF & Multidisciplinar & Turismo e Sociedade \\
\hline
\end{tabular}

Fonte: Elaboração própria a partir dos dados coletados. 
Comparando a segunda e a terceira coluna da tabela acima, é possível identificar um alinhamento dos resultados encontrados pela nuvem de palavras e o que é informado como áreas de concentração do programa. O resultado mais destoante fica com o IFS, visto que em sua nuvem de palavras os termos acessibilidade e território remetem as questões do Planejamento Territorial, mas a página do programa indica concentração de pesquisas na área de Gestão do Turismo.

\section{Considerações finais}

Embora o primeiro curso de mestrado em Comunicação, com uma linha em Lazer e Turismo tenha sido implementado em 1993 pela Escola de Comunicação e Artes da Universidade de São Paulo (ECA-USP), o mestrado mais antigo ainda em funcionamento hoje no Brasil é da UNIVALI, cujo inicio se deu 1997. Sendo desse mesmo programa o primeiro curso de doutorado em Turismo e Hotelaria iniciado apenas em 2013.

A consolidação da área do Turismo vem se fortalecendo ao longo dos anos, com uma concentração de abertura de cursos de pós-graduação, de veiculação de periódicos específicos da área, assim como, a criação da Associação Nacional de Pesquisadores de Turismo (ANPTUR), a partir dos anos 2000.

Os estudos acerca do tema da produção do conhecimento no Turismo em nível stricto sensu também são recentes, compreendem uma jornada de pouco mais de 25 anos ao considerar que o estudo pioneiro defendido em sua tese por Rejowski ocorreu em 1993, o qual posteriormente inspirou uma série de estudos.

Com o objetivo de apresentar um cenário panorâmico do perfil de pós-graduação em turismo no Brasil adotou-se uma postura investigativa ostensiva, pesquisando não uma amostra, mas o universo de docentes que estão vinculados a estes programas. Observou-se que apesar das formações em graduação, mestrado e doutorado serem múltiplas (no caso de mestrado e doutorado mais de 50 diferentes) ao utilizar uma macroclassificação de grandes áreas da CAPES, verificou-se que há uma concentração no Colégio de Humanidades.

Portanto, pode-se concluir que a grande produção de estudos e pesquisas qualitativas em território nacional tem uma possível relação direta com essa formação concentrada.

No que diz respeito quantitativamente à produção acadêmica: USP, UFRN, UCS e Univali têm se destacado nos últimos anos. No quesito de produção internacional, em números relativos baseados nas produções sinalizadas pelos docentes como mais relevantes têm-se que a USP, UCS e UFRN despontam como as instituições que mais produzem internacionalmente.

Por fim, ao analisar as áreas de interesse de todos os docentes, descobriu-se que além do turismo, há também disposição para pesquisas em hospitalidade, lazer, gestão, políticas públicas e meio-ambiente. $\mathrm{E}$ ao destrinchar essa análise por instituição pode-se notar os interesses específicos que desenham um perfil que quase sempre se encaixa com a descrição das áreas de concentração dos programas de pós-graduação. É o caso, por exemplo, da Univali que, pela análise qualitativa utilizada, apresentou interesses em gestão, marketing e gastronomia que parece sincronizar com a área de concentração do programa "Planejamento e Gestão do Turismo e Hotelaria".

De maneira geral, esse artigo desenhou um panorama da pesquisa cientifica em turismo no Brasil, se manifestando no sentido de ser um diagnóstico inicial. Dessa forma, indica-se que futuras pesquisas abordem a temática a partir de estudos comparativos com outras áreas próximas do turismo e até mesmo com panoramas internacionais. Assim como sobre o tema da internacionalização da área.

\section{Bibliografia}

Brasil. 1965. Parecer no 977/65, C.E.Su, aprov. em 3-12-65. Definição Dos Cursos De Pós-Graduação. 11. Brasil. 2019. Qual a diferença entre pós-graduação lato sensu e stricto sensu? Retrieved October 18, 2019, from Ministério da Educação website: http://portal.mec.gov.br/component/content/article?id=13072:qual-a-diferenca-entre-pos-graduacao-lato-sensu-e-stricto-sensu

CAPES. 2017. Resultado final da Avaliação Quadrienal 2017. Retrieved June 30, 2020, from Coordenação de Aperfeiçoamento de Pessoal de Nível Superior website: https://www.capes.gov.br/36-noticias/8691-capes-divulga-resultado-final-da-avaliacao-quadrienal-2017

CAPES. 2019. Qualis Periódicos e classificação de produção intelectual. Retrieved October 18, 2019, from Coordenação de Aperfeiçoamento de Pessoal de Nível Superior website: https://www.capes.gov. br/avaliacao/instrumentos-de-apoio/qualis-periodicos-e-classificacao-de-producao-intelectual 
CAPES. 2020. Avaliação Quadrienal. Retrieved June 30, 2020, from Coordenação de Aperfeiçoamento de Pessoal de Nível Superior website: http://avaliacaoquadrienal.capes.gov.br/

Ciências sem Fronteiras. 2020. Pós-Doutorado. Retrieved March 20, 2020, from http://www.cienciasemfronteiras.gov.br/web/csf/pos-doutorado

Freitas, C. C. da S. 2018. O discurso epistemológico sobre turismo e sua transposição nos programas de pós-graduação do Brasil (Universidade de Brasília, Mestrado Profissional em Turismo). https:// doi.org/10.1017/CBO9781107415324.004

Holanda, L., Widmer, G., \& Leal, S. 2014. A produção científi ca em turismo no Brasil: reflexões e proposições a partir de um estudo revisional. Anais Brasileiros de Estudos Turrísticos: ABET, 4(1), 72-79.

Leal, S. R. 2011. Pesquisa em Turismo no Brasil: uma Revolução Silenciosa? Turismo \& Sociedade, 04(84), 144-147.

Rejowski, M., \& Barbanti, C. H. 2019. Construção de um Tesauro Brasileiro de Turismo. Revista Turismo Em Análise, 29(2), 182-195. https://doi.org/10.11606/issn.1984-4867.v29i2p182-195

Rejowski, M., \& Kobashi, N. Y. 2011. Subsídios para elaboração de um Tesauro Brasileiro de Turismo. Revista Turismo Em Análise, 22(3), 579. https://doi.org/10.11606/issn.1984-4867.v22i3p579-598

Santos, G. E. de O., \& Rejowski, M. 2013. Comunicação científica em turismo no Brasil: Análises descritivas de periódicos nacionais entre 1990 e 2012. Revista Brasileira de Pesquisa Em Turismo, 7(1), 149-167. https://doi.org/10.7784/rbtur.v7i1.578

Sogayar, R. L., \& Rejowki, M. 2011. Ensino Superior em Turismo em busca de novos paradigmas educacionais: problemas, desafios e forças de pressão. Revista Turismo Visão e Ação, 13(3), 282-298.

Velloso, A. (2014). A pós-graduação no Brasil : legados e desafios. Almanaque Multidisciplinar De Pesquisa, 1(1), 4-15. Retrieved from http://publicacoes.unigranrio.edu.br/index.php/amp/article/ viewFile/2648/1241

Notas

O parecer foi emitido por Newton Lins Buarque Sucupira.

Fundação vinculada ao Ministério da Educação.

Regulamentado pela Portaria MEC N 389, de 23 de março de 2017 e pela Portaria CAPES No 131, de 28 de junho de 2017 (Brasil, 2019).

4 Ferramenta para coletar informações, realizar análises e avaliações. É a base de referência do Sistema Nacional de Pós-Graduação do Brasil. 